\title{
OBSERVACִÕES SOBRE A BIOLOGIA DE APIOMERUS LANIPES (FABRICIUS) (HEMIPTERA, REDUVIIDAE)
}

\author{
Benedicto Ferreira do Amaral Filho ${ }^{1}$ \\ Ismael Gióia ${ }^{1}$ \\ Claudia Maria Waib ${ }^{2}$ \\ Elaine Mendeleck ${ }^{2}$ \\ Fernando Luis Cônsoli ${ }^{3}$
}

\begin{abstract}
OBSERVATIONS ON THE BIOLOGY OF APIOMERUS LANIPES (FABRICIUS) (HEmIPTERA, REDUVIIDAE). The aim of the present work is to describe the biological cycle of the Apiomerus lanipes (Fabricius, 1803) (Hemiptera, Reduviidae) on laboratory conditions. Adults of Drosophila sp. (Diptera, Drosophilidae) and Ceratitis capitata (Wiedemann, 1824) (Diptera, Tephritidae) was offered for bug feeding (nymphs and adults). The developmental of the entire cycle (egg to adult) was 288.26 days and 294.85 days for males and females, respectively. The duration of egg stage and nymphal stage was 23.5 days and 269.02 days, respectively.

KEY WORDS. Reduviidae, biological cycle, laboratory conditions
\end{abstract}

Os reduvídeos predadores são insetos considerados de grande importância econômica, por serem agentes controladores de pragas agrícolas (BUENO \& BERTI FILHO 1991). Eles matam sua presa prendendo-a com suas fortes pernas e então inserem seus estiletes para sugar sua hemolinfa. Espécies dos gêneros Reduvius Fabricius, 1775, Zelus Fabricius, 1803 e Apiomerus Hahn, 1831, são exemplos de predadores desta família e têm muito em comum. Eles podem adquirir hábitos canibalísticos quando há redução na oferta de alimento. Isto requer uma abundância de presas vivas quando são criados em laboratório (RICKMAN \& RYCKMAN 1968).

As espécies de Apiomerus são consideradas predadoras eficazes de insetos. Em alguns casos, podem ser consideradas nocivas por atacarem insetos úteis como as abelhas. Contudo, quando se trata de insetos pragas, como o percevejo do arroz Mormidea poecila Dallas, 1851 (Hemiptera, Pentatomidae), são tidas como vantajosas (CosTA LimA 1940).

AMARAL (1982) em experimentos em campos de soja, observou a presença de espécies de Apiomerus atacando Anticarsia gemmatalis Hübner, 1818 (Lepi-

1) Departamento de Zoologia, Instituto de Biologia, Universidade Estadual de Campinas, Caixa Postal 6109, 13083-970 Campinas, São Paulo, Brasil.

2) Pós-graduação em Parasitologia, Instituto de Biologia, Universidade Estadual de Campinas, Caixa Postal 6109, 13083-970 Campinas, São Paulo, Brasil.

3) Pós-graduação em Entomologia, Escola Superior de Agronomia Luiz de Queiroz, Universidade de São Paulo, Caixa Postal 09, 13400-000 Piracicaba, São Paulo, Brasil. 
doptera, Noctuidae). DE LOYA (1987) observou a predação de coleópteros adultos por Apiomerus venosus Stål, 1872 e Apiomerus pictipes Herrich-Schaeffer, 1848. ReIS \& SOUZA (1993) registraram a presença de Apiomerus sp. em armadilhas para adultos da broca-da-bananeira, Comopolites sordidus Germar, 1824 (Coleoptera, Curculionidae) no campo.

ZANúNCIO et al. (1992) estudaram métodos para criação de hemípteros predadores de largatas, dentre eles Apiomerus sp.. Eles ressaltam o potencial predatório destes insetos em surtos de lagartas desfolhadoras de eucalipto.

As informações disponíveis na literatura sobre o gênero Apiomerus são limitadas, sendo voltadas para registro e ocorrência.

Dados sobre aspectos biológicos são extremamente importantes para o estudo destes hemípteros predadores como agentes em potencial para o manejo integrado de pragas.

O objetivo do presente trabalho é estudar a biologia de A. lanipes (Fabricius, 1803) sob condições de laboratório, contribuindo assim com informações básicas e fundamentais para uma possível utilização deste inseto em projetos de manejo integrado.

\section{MATERIAL E MÉTODOS}

A criação de A. lamipes foi desenvolvida no Laboratório de Criação de Insetos do Departamento de Zoologia - IB/UNICAMP, com fotoperíodo determinado ( 12 horas de fotofase) e sob condições de temperatura e umidade relativa ambientes $\left(X_{\mathrm{t}}=26,25 \pm 2,0^{\circ} \mathrm{C} ; \mathrm{X}_{\mathrm{UR}}=77,07 \pm 10,00 \%\right.$ U.R. $)$.

Adultos de A. lanipes foram coletados na região de Campinas e de Santa Maria da Serra (São Paulo), inciando-se uma criação estoque da qual foi retirada uma amostra de 72 ovos para o acompanhamento do ciclo biológico.

As ninfas de primeiro e segundo estádios foram mantidas em frascos de vidro de $1,5 \mathrm{~cm}$ de diâmetro por $4,5 \mathrm{~cm}$ de altura, tampados com algodão hidrófilo. A partir do terceiro estádio ninfal até o estágio de adulto, os insetos foram mantidos em frascos de vidro de $5,0 \mathrm{~cm}$ de diâmetro por $6,5 \mathrm{~cm}$ de altura, fechados com tecido de algodão e forrados com papel absorvente. Adotou-se, para todos os estádios, a metodologia de criar as ninfas individualmente.

Os casais formados a partir da emergência dos adultos foram mantidos em frascos de vidro de $7,5 \mathrm{~cm}$ de diâmetro por $10,5 \mathrm{~cm}$ de altura (um casal por frasco).

A higienização dos frascos cativeiros foi realizada em dias alternados.

Foram oferecidos adultos vivos de Drosophila sp. (Diptera, Drosophilidae) para as ninfas de primeiro a terceiro estádios, na proporção de 5, 8, e 12 moscas por ninfa, respectivamente. Tanto para as ninfas de quarto e quinto estádios como para adultos foram oferecidos adultos vivos de Ceratitis capitata (Wiedemann, 1824) (Diptera, Tephritidae), na proporção de 7, 12 e 15 moscas por indivíduo, respectivamente. As moscas foram oferecidas aos hemípteros em dias alternados e a escolha de duas fontes alimentares diferentes baseou-se diretamente na proporção de tamanho predador $\mathrm{x}$ presa. 
A criação estoque de Drosophila sp. foi mantida no próprio laboratório em dieta artificial, enquanto os adultos de $C$. capitata foram cedidos periodicamente pelo Departamento de Genética e Evolução - IB/UNICAMP.

Larvas vivas de quartos estádio de Spodoptera frugiperda (Smith, 1797) (Lepidoptera, Noctuidae) foram oferecidas para algumas ninfas de quinto estádio de $A$. lanipes, a fim de avaliar a aceitação do reduvídeo para outro tipo de dieta. As observações dos aspectos biológicos de A. lanipes foram realizadas diariamente.

A razão sexual dos adultos de $A$. lanipes foi calculada segundo SILVEIRA NETO et al. (1976).

\section{RESULTADOS E DISCUSSÃO}

\section{ESTÁGIO DE OVO}

Os ovos de $A$. lanipes possuem o formato de um cilindro alongado e são colocados em grupos. O número máximo de ovos agrupados observado foi de 44 , sempre aderidos ao substrato e entre si.

O período médio de incubação dos ovos foi de 23,5 dias (amplitude 23-24) com uma viabilidade máxima de $95,83 \%$.

SWADENER \& YONKE (1973), em estudo com A. crassipes (Fabricius, 1803), observaram no estágio de ovo um período médio de 16,7 dias (amplitude 14-20), para uma temperatura de 21 e $29^{\circ} \mathrm{C}$ (noite e dia) e uma umidade relativa variando de 40 a $50 \%$ durante o dia e de $50 \mathrm{a} 60 \%$ à noite.

\section{ESTÁGIO DE NIFA}

A duração em dias de cada estádio ninfal, assim como a duração total do estágio de ninfa e respectivas taxas de mortalidade estão descritas na tabela I.

Tabela I. Duração (dias) e mortalidade (\%) dos estádios ninfais de Apiomerus lanipes (T=26,25 $2,00^{\circ}$ C; U.R. $=77,07 \pm 10,00 \%$; fotofase 12 horas).

\begin{tabular}{lcccccc}
\hline & \multicolumn{5}{c}{ Estádios } & \multicolumn{2}{c}{$\begin{array}{c}\text { Estágio de ninfa } \\
\text { completo }\end{array}$} \\
\cline { 2 - 6 } & $1^{\circ}$ & $2^{\circ}$ & $3^{\circ}$ & $4^{\circ}$ & $5^{\circ}$ & \\
\hline$N^{\circ}$ observado & 52 & 52 & 48 & 47 & 35 & 35 \\
Amplitude & $27-49$ & $30-59$ & $28-59$ & $22-65$ & $39-131$ & $201-329$ \\
Média & 37,69 & 42,82 & 40,83 & 37,06 & 101,22 & 269,02 \\
Desvio padrão & 5,12 & 7,20 & 6,39 & 8,87 & 24,25 & 22,53 \\
Mortalidade & 1,88 & 0,00 & 7,69 & 2,08 & 25,53 & 33,96 \\
\hline
\end{tabular}

Pode-se notar que o quinto estádio foi o mais longo (101,22 dias), e no qual houve um maior índice de mortalidade $(25,53 \%)$. Nos demais estádios ninfais o índice de mortalidade não passou de 7,69\%.

A duração do estágio de ninfa obtida para outros reduvídeos predadores criados em condições de laboratório foram 123,8 dias, com alta taxa de mortalidade 
entre os estádios, para $A$. crassipes mantido por SWADENER \& YONKE (1973) com larvas de diversos lepidópteros; tempos de 8,$6 ; 7,4 ; 12,6 ; 12,2 ; 14,2$ dias respectivamente para o primeiro ao quinto estádio de Zelus leucogrammus (Pertry, 1834) (НАвIB 1976); 72,86 dias para Montina confusa (Stål, 1859) criado por BUENO \& BERTI FILHO (1984) com larvas de Lepidoptera; tempos de 89,87; 67,97; 72,74 e 66, 18 dias para esta mesma espécie mantida com quatro diferentes dietas por CôNSOLI \& AMARAL FILHO (1992).

Com relação às ninfas de quinto estádio alimentadas com larvas de $S$. frugiperda, foi observada uma boa aceitação deste tipo de dieta. As ninfas de $A$. lanipes chegaram a predar larvas de último estádio de $S$. frugiperda, as quais são grandes e ágeis (Fig. 1).

Um dos principais fatores responsáveis pela mortalidade observada no presente trabalho pode estar relacionado com anormalidades que ocorrem com o inseto no momento da ecdise, fato relatado para outros reduvídeos (CônSOLI \& Amaral Filho 1992; VenNison \& AMBROSE 1989).

\section{ESTÁGIO ADULTO}

Nos 35 adultos de $A$. lanipes obtidos, pôde-se verificar uma razão sexual de 0,57 .

Os dados referentes às atividades reprodutivas dos adultos em laboratório e sua longevidade (em dias) estão representados na tabela II.

Tabela II. Atividades reprodutivas (periodos em dias) e longevidade (dias) de Apiomertus lanipes, obtidas $\mathrm{em}$ laboratório ( $\mathrm{T}=26.25 \pm 2.00^{\circ} \mathrm{C}$; U.R. $=77.07 \pm 10.00 \%$ : fotofase 12 horas).

\begin{tabular}{lcccccccc}
\hline & \multirow{2}{*}{$\begin{array}{c}N^{\circ} \text { de } \\
\text { ovos/fêmea }\end{array}$} & \multicolumn{2}{c}{ Atividades reprodutivas } & & \multicolumn{2}{c}{ Longevidade } \\
\cline { 3 - 4 } & & Pré-ovip. & Ovip. & Pós-ovip. & & Macho & Fêmea \\
\hline Média & 29,66 & 70,16 & 1 & 3,16 & & 47,80 & 72,25 \\
Desvio & 29,52 & 6,14 & - & 2,78 & & 9,74 & 7,29 \\
Amplitude & $2-73$ & $62-80$ & 1 & $1-8$ & & $2-143$ & $12-144$ \\
\hline
\end{tabular}

O ciclo total de ovo a adulto foi de 288,26 dias (amplitude 224-313) para os machos, e de 294,85 dias (amplitude de 223-352) para as fêmeas.

Embora cada espécie tenha um ciclo biológico característico, a duração das diferentes etapas desse ciclo podem sofrer variações consideráveis, particularmente em insetos criados sob condições de laboratório. Fatores como tipo de dieta oferecida, volume do frasco onde o inseto é criado, condições climáticas, etc., exercem forte influência sobre o seu ciclo biológico.

AGRADECIMENTOS. Ao Conselho Nacional de Desenvolvimento Científico e Teenológico (CNPq) e Fundo de Apoio ao Ensino e Pesquisa (FAEP/UNICAMP), pelo apoio financeiro para a realização deste trabalho. Ao Departamento de Genética e Evolução - IB/UNICAMP, pelo fornecimento semanal de adultos de Ceratitis capilata. 


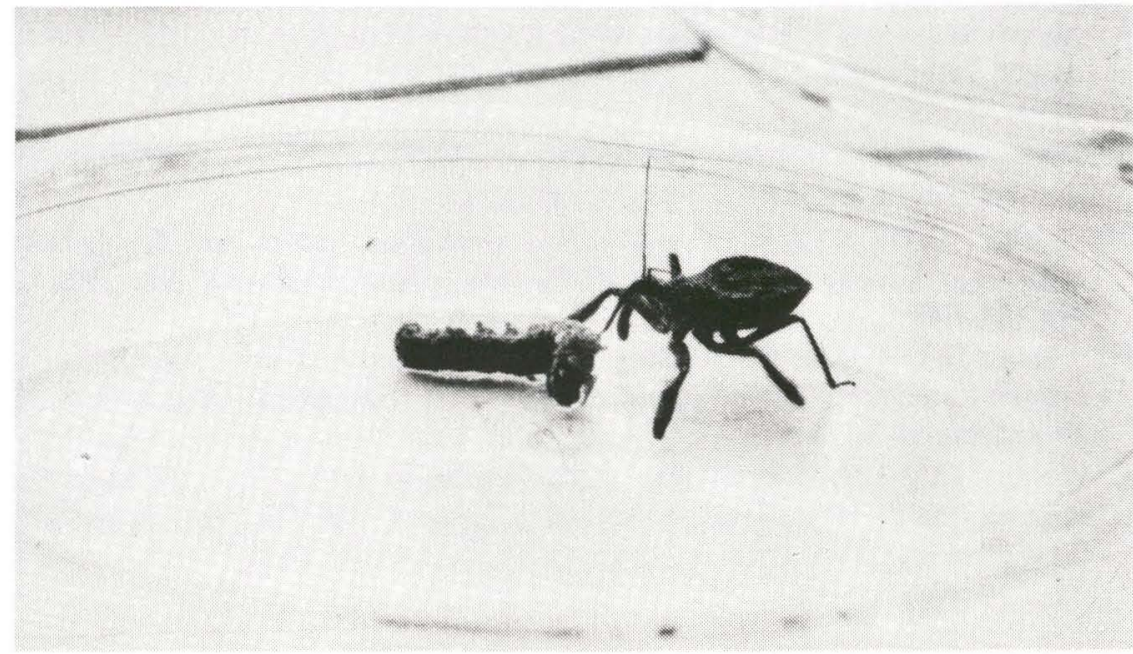

Fig. 1. Ninfa de quinto estádio de Apiomerus lanipes predando larva de último estádio de Spodoptera frugiperda.

\section{REFERÊNCIAS BIBLIOGRÁFICAS}

Amaral, M.E.C. 1982. Controle biológico natural e aplicado de Anticarsia gemmatalis Hübner, 1818 (Lepidoptera, Noctuidae) em campos de soja. Tese de Mestrado, não publicada, Instituto de Biologia, Universidade Estadual de Campinas, Campinas, 198p.

Bueno, V.H.P. \& E. BerTi Filho. 1984. Montina confusa (Stål, 1859) (Hemiptera: Reduviidae: Zelinae): I. Aspectos biológicos. Revta bras. Ent. 28 (3): 345-353.

. 1991. Controle biológico de insetos com predadores. Inf. Agropec. 15 (167): 41-52.

Cônsoli, F.L. \& B.F. Amaral Filho. 1992. Ciclo biológico de Montina confusa (Stål, 1859) (Hemiptera, Reduviidae) alimentado com diferentes presas. Revta bras. Ent. 36 (3): 697-702.

Costa Lima, A. 1940. Insetos do Brasil: Hemípteros. Rio de Janeiro, Esc. Nac. Agron., 2, $351 \mathrm{p}$.

DE LOYA, C. 1987. Observaciones acerca de la depredacion de Apiomerus venosus y A. pictipes (Hemiptera: Reduviidae) sobre coleopteros lamelicornios adultos. Folia Entomol. Mex. 71: 65-66.

HABIB, M.E.M. 1976. Estudos biológicos sobre Zelus leucogrammus Perty, 1834 (Hemiptera: Reduviidae: Zelinae). An. Soc. ent. Brasil 5 (2): 120-129.

ReIS, P.R. \& J.C. DE SouZA. 1993. Atratividade de dois tipos de iscas sobre adultos da broca-da-bananeira e influência no controle biológico. Resumos XIV Congresso Brasileiro de Entomologia, Piracicaba, Soc. Ent. Brasil, p.312. 
RYCKMAN, R.E. \& A.E. RYCKMAN. 1968. Reduviid Bugs. In: C.N. SMITH (ed.).

Insect colonization and mass production. NY \& Londres, Academic Press, $618 \mathrm{p}$.

Silveira Neto, S; O. Nakano; D. Barbin \& N. A. Villa Nova. 1976. Manual de Ecologia de Insetos. São Paulo, Ed. Agron. Ceres, 419p.

SWADENER, S.D. \& T.R. YonKE. 1973. Immature stages and biology of Apiomerus crassipes (Hemiptera: Reduviidae). Ann. Entomol. Soc. Amer. 66: 188-196.

VENNISON, S.J. \& D.P. AMBrose. 1989. Biology and predatory potential of a reduviid predator, Oncocephalus annulipes Stål (Hemiptera: Reduviidae). J. Biol. Control 3: 24-27.

Zanúncio, J.C.; J.B.C. Alves; R.C. Sartório \& J.E. M. Leite. 1992. Métodos para criação de hemípteros predadores de lagartas. An. Soc. ent. Brasil 21: 245-251.

Recebido em 30.VIII.1993; aceito em 13.V.1994 